\title{
HOST-SPECIFIC IN VITRO COLONISATION OF FISH EPITHELIA BY GYRODACTYLIDS
}

\author{
Thomas B. LARSEN, Kurt BUCHMANN* \\ Department of Veterinary Pathobiology, Section of Fish Diseases, Royal Veterinary and Agricultural University, \\ Frederiksberg, Copenhagen, Denmark
}

Larsen T.B., Buchmann K. 2006. Host-specific in vitro colonisation of fish epithelia by gyrodactylids. Acta Ichthyol. Piscat. 36 (2): 113-118.

\begin{abstract}
Background. The monogeneans, Gyrodactylus derjavini Mikailov, 1975 and G. salaris Malmberg, 1957, are specific parasites of brown trout, Salmo trutta L., and Atlantic salmon, S. salar L., respectively. Rainbow trout, Oncorhynchus mykiss (Walbaum, 1792), is also susceptible to G. derjavini but, similarly like brown trout, mounts a host response against this parasite. Rainbow trout can also experience infections with $G$. salaris but experiences generally lower infection levels compared to Atlantic salmon. This in vitro work was conducted in order to elucidate if the parasites could find and select specific host scales without influence of live and mobile hosts.

Material and Methods. Using these two parasite species, a series of in vitro studies on host specificity were conducted. Scales with intact epithelia from rainbow trout, carp and salmon were cultivated and the two congeneric gyrodactylids (Gyrodactylus derjavini and G. salaris) were allowed to colonise the scales. Parasites attaching to the different scale types were monitored over $24 \mathrm{~h}$.

Results. The gyrodactylids colonised fish scales to a lesser extent, in vitro, compared to experiments where live fish were exposed to parasites. However, the parasites attached to a higher extent to in vitro-cultured fish scales with epithelia from their specific hosts: $G$. salaris relocated more often to salmon scales compared to the scales from rainbow trout and common carp, Cyprinus carpio L. In contrast, G. derjavini colonised preferentially cultured scales from rainbow trout and, to a lesser degree, scales from salmon.

Conclusion. The work indicated that gyrodactylids colonising host epithelia are at least partly dependent on host movements but also have an ability to sense host-specific molecules due to their specific attachment to specific host scales.
\end{abstract}

Keywords: Gyrodactylus derjavini, G. salaris, host specificity, epithelium, in vitro, rainbow trout, carp, salmon, fish

\section{INTRODUCTION}

Host specificity of monogeneans is a well-described biological factor, which plays a major role in their ecology and population biology (Malmberg 1993, Whittington et al. 2000, Bakke et al. 2002, Buchmann and Lindenstrøm 2002). Two of the parasite-host systems exhibiting a pronounced predilection of specific monogeneans for certain hosts are the association between G. derjavini Mikailov, 1975 and the brown trout, $S$. trutta L., and the association between G. salaris Malmberg, 1957 and the Atlantic salmon, $S$. salar $\mathrm{L}$. The rainbow trout, $O$. mykiss (Walbaum, 1792), was introduced into Northern Europe, which is an endemic area for $G$. derjavini, within the last 130 years. However, the latter host shows interactions with G. derjavini corresponding to the relations between brown trout and this gyrodactylid (Buchmann and Uldal 1997). Further, it was recently demonstrated experimentally that $G$. derjavini preferentially colonised rainbow trout to Atlantic salmon in a multiple-choice test and that $G$. salaris selected Atlantic salmon over rainbow trout when given the choice (Buchmann et al. 2004). These parasite-host associations are reflected under natural conditions. Thus, in endemic areas, brown trout are primarily infected with $G$. derjavini while salmon generally harbour $G$. salaris (cf. Mo 1991, 1993, 1997, Bakke et al. 2002). However, the basis for this specific host selection is not well elucidated. Chemical, mechanical, and behavioural mechanisms have been suggested to explain this host specificity (Whittington et al. 2000, Buchmann and Lindenstrøm 2002). Although chemical host substances are considered to offer a good explanation both among gyrodactylids (Buchmann 2001, Jørndrup and Buchmann 2005) and among other monogeneans (Yoshinaga et al. 2000, 2002, Hirazawa et al. 2003), it cannot be excluded that a range of abiotic and 
biotic factors (Kearn 1967) could also induce host colonisation. The present study was conducted on colonisation of $G$. salaris and $G$. derjavini of in vitro-cultured host epithelia in order to exclude other factors from the analysis.

\section{MATERIALS AND METHODS}

Fish: Eggs of Atlantic salmon, S. salar (from the eas Atlantic Scottish river Conon stock), and rainbow trout, O. mykiss (from the Danish trout farm Faarup mølle in Jutland), were hatched and reared at the salmon hatchery of Bornholm (Denmark). Following disinfection in iodophore (Actomar K30) the eggs were incubated in hatchery trays and hatched in municipal freshwater at $\mathrm{pH} 7-8$ and at a temperature of $7^{\circ} \mathrm{C}$. The fish were fed commercial pelleted fish feed (BioMar A/S, Denmark) at $10^{\circ} \mathrm{C}$ during the pre-experimental period. Common carp, C. carpio L., fry was obtained from a laboratory stock (Wageningen University, the Netherlands). All fish were transported to a pathogen-free university system (KVL, Frederiksberg, Denmark) and kept in recirculated water at least 2 months prior to experimentation.

Experimental temperature: All experiments were conducted at $12-13^{\circ} \mathrm{C}$ in a temperature-controlled room with constant artificial illumination.

Fish epithelia on scales for in vitro culture: Epithelium-covered scales were obtained from salmon (age $2+$ ), rainbow trout (age $2+$ ), and common carp (age $2+$ ).

Parasites: A population of $G$. salaris, originating from the Norwegian river Laerdalselva, was imported to Denmark and kept on susceptible Scottish (Conon River) salmon, $S$. salar (age 1+), in recirculated water, in an isolated experimental fish tank unit, for two years before the experiment began. The $G$. derjavini population was collected on a Danish trout farm (Paelebro, Dambrug) and kept on rainbow trout $O$. mykiss (age $1+$ ) in recirculated water for seven years before experimental start.

Isolation of parasites: Gyrodactylids were isolated by cutting heavily infected fins and placing these into tapwater filled Petri dishes. Parasites leaving the fins voluntarily, within $60 \mathrm{~min}$, were then used for further experimentation without manipulating the worms with any tools.

In vitro cultivation of epithelial cells on scales: Scales from each species were removed with fine forceps from the dorsal region of the fish and rinsed in Hanks Balanced Salt Solution with a salinity of $0.9 \%$ (HBSS 14175 053, Invitrogen, UK) in Petri dishes (diameter $60 \mathrm{~mm}$ ) for $15 \mathrm{~min}$. Subsequently, the scales were transferred to tissue-culture medium L15 (Sigma L5520, USA) supplemented with antibiotics penicillin/streptomycin solution (penicillin $250 \mathrm{u} \cdot \mathrm{mL}^{-1}$, streptomycin $250 \mu \mathrm{g} \cdot \mathrm{mL}^{-1}$ ) (Sigma P0781, USA). This culture medium was diluted ( $1: 8)$ using sterile water. After $24 \mathrm{~h}$ of pre-incubation, the scales were placed in groups in a Petri dish (60 mm diameter) with freshly diluted (1:8) L15 tissue-culture medium (Sigma L5520) and a normal atmosphere over the culture medium. The epithelia on scales were regularly checked (light microscope, magnification $40-100 \times$ ) to ensure their integrity.

\section{Parasite colonisation of epithelia in vitro}

Experimental protocol: Two experiments were conducted under the dissection microscope (magnification $7-40 \times)$.

1) The first experiment was made with one type of scales (10 scales) in each Petri dish, either scales from Atlantic salmon (average scale size: $9.9 \mathrm{~mm}^{2}$ ), rainbow trout (a-verage scale size: $7.4 \mathrm{~mm}^{2}$ ), or carp (average scale size: $8.5 \mathrm{~mm}^{2}$ ). Scales were placed in the periphery of the Petri dish. Following 24-h pre-incubation of scales, a number (9-15) of live specimens of G. salaris or G. derjavini was added to the centre $\left(1 \mathrm{~cm}^{2}\right)$ of each Petri dish. The transfer of parasites from the isolation dish to the experimental dish was done by tilting the dish (resulting in the gyrodactylids concentrating in one pole of the dish) and by the use of a preparation needle. In order to decrease manipulation of the parasites they were then allowed to migrate from the isolation dish to the experimental dish voluntarily but they were guided by the preparation needle under the dissection microscope. Their numbers, however, were always watched to fit within the range of 9-15. Nine replicates were prepared for each scale type and each parasite species. The number of parasites attached to the scales was recorded at $1,2,3,4,5,6$, and $24 \mathrm{~h}$ post-exposure.

2) In the second experiment three epithelium-covered scales from each fish species (salmon, rainbow trout, carp) (total of nine scales) were placed in the same Petri dish. The scales were distinguished based on morphology. Nine to 15 specimens of G. salaris or G. derjavini were added to the dish and the numbers of attached parasites were recorded as in experiment 1 .

Calculations and statistics: Within each of the different colonisation experiments the testing of parasite attachment the non-parametric Kruskal-Wallis test and Dunn's test were used to detect differences between experimental groups. The probability level was set at 5 percent in all tests.

\section{RESULTS}

G. salaris and G. derjavini colonised epithelium-covered scales with a maximum rate of relocation (from the Petri dish to fish scale) of $34 \%$ (minimum rate $1.4 \%$ ). Some of the parasites died during incubation, whereas others released their embryos (Table 1). However, when attached $G$. salaris found scales from $S$. salar significantly more often $(P<0.05)$ than scales from rainbow trout and carp (Figs. 1, 2). After $1 \mathrm{~h}$, more than $50 \%$ of all relocated $G$. salaris were found on salmon scales. This initial preference of $G$. salaris for $S$. salar increased in subsequent hours. Correspondingly, G. derjavini colonised scales from rainbow trout more often than salmon and carp scales $(P<0.05)$, both in single- and multiple-choice situations (Figs. 1, 2). Generally, it was observed that both $G$. salaris and G. derjavini, at least to some extent, would colonise scales from other hosts than their preferred ones (Tables 1, 2; Figs. 1, 2). The total colonisation rate did not increase when scales from the three different host species were exposed together. Thus maximum colonisation rates were between $16 \%$ and $18 \%$ in these cases (Table 3 ). 
Gyrodactylus salaris experiment with separate scales from salmon, rainbow trout, and carp; summary of 9 Petri dishes

\begin{tabular}{llccccccc}
\hline & \multicolumn{7}{c}{ Hours post exposure } \\
\cline { 3 - 9 } & & 1 & 2 & 3 & 4 & 5 & 6 & 24 \\
\hline Separate & No. of parasites used & 114 & 110 & 106 & 111 & 101 & 92 & 72 \\
salmon & No. of parasites attached & 18 & 24 & 27 & 29 & 26 & 31 & 17 \\
scales & \% attached & 15.8 & 21.8 & 25.5 & 26.1 & 25.7 & 33.7 & 23.6 \\
Separate & No. of parasites used & 102 & 99 & 101 & 95 & 98 & 89 & 71 \\
rainbow & No. of parasites attached & 6 & 13 & 7 & 6 & 5 & 8 & 1 \\
trout scales & \% attached & 5.9 & 13.1 & 6.9 & 6.3 & 5.1 & 9.0 & 1.4 \\
Separate & No. of parasites used & 109 & 107 & 101 & 96 & 97 & 98 & 60 \\
carp & No. of parasites attached & 8 & 6 & 6 & 6 & 9 & 13 & 2 \\
scales & \% attached & 7.3 & 5.6 & 5.9 & 6.3 & 9.3 & 13.3 & 3.3 \\
\hline
\end{tabular}

Table 2

Gyrodactylus derjavini experiment with separate scales from salmon, rainbow trout, and carp; summary of 10 Petri dishes for each species

\begin{tabular}{|c|c|c|c|c|c|c|c|c|}
\hline & \multicolumn{7}{|c|}{ Hours post exposure } \\
\hline & & 1 & 2 & 3 & 4 & 5 & 6 & 24 \\
\hline Separate & No. of parasites used & 94 & 89 & 70 & 56 & 55 & 51 & 38 \\
\hline salmon & No. of parasites attached & 4 & 12 & 8 & 6 & 6 & 7 & 5 \\
\hline scales & $\%$ attached & 4.3 & 13.4 & 11.4 & 10.7 & 10.9 & 13.7 & 13.1 \\
\hline Separate & No. of parasites used & 84 & 62 & 50 & 42 & 39 & 35 & 38 \\
\hline rainbow & No. of parasites attached & 5 & 7 & 8 & 6 & 8 & 6 & 8 \\
\hline trout scales & $\%$ attached & 5.9 & 11.3 & 16.0 & 14.3 & 20.5 & 17.1 & 21.1 \\
\hline Separate & No. of parasites used & 99 & 69 & 59 & 53 & 51 & 43 & 47 \\
\hline carp & No. of parasites attached & 3 & 5 & 8 & 5 & 1 & 3 & 3 \\
\hline scales & $\%$ attached & 3.0 & 7.2 & 13.5 & 9.4 & 2.0 & 7.0 & 6.3 \\
\hline
\end{tabular}

Table 3

Colonisation experiments with G. salaris or G. derjavini and using scales from three host species (salmon -3 scales, rainbow trout -3 scales, and carp -3 scales) in each Petri dish; summary of 10 Petri dishes

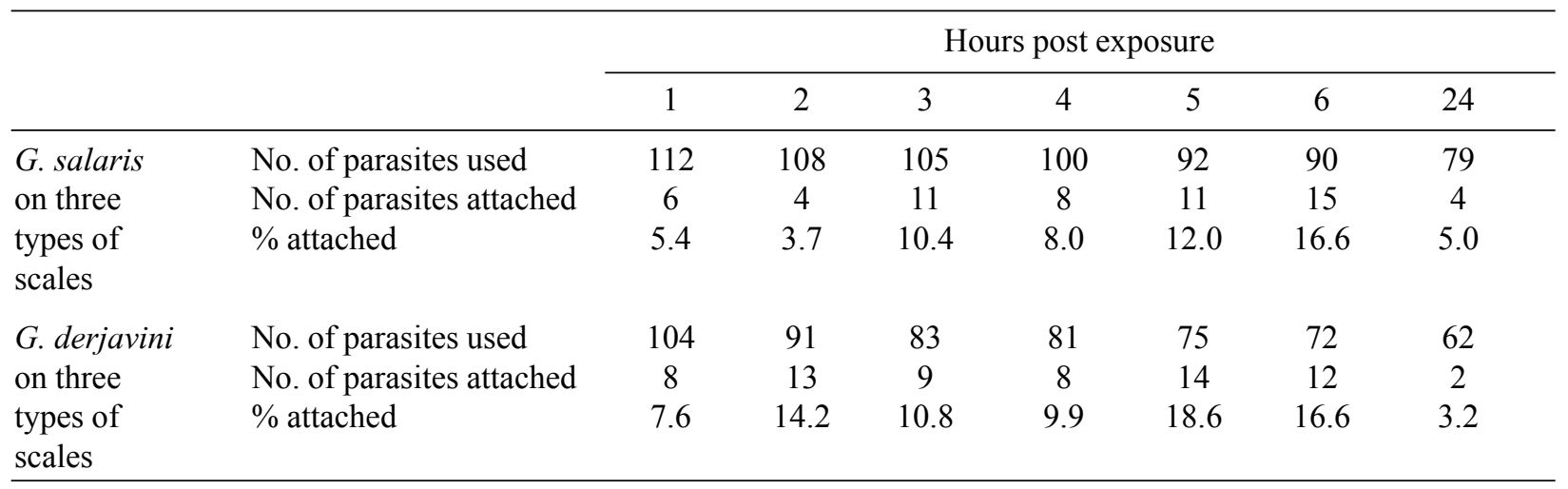




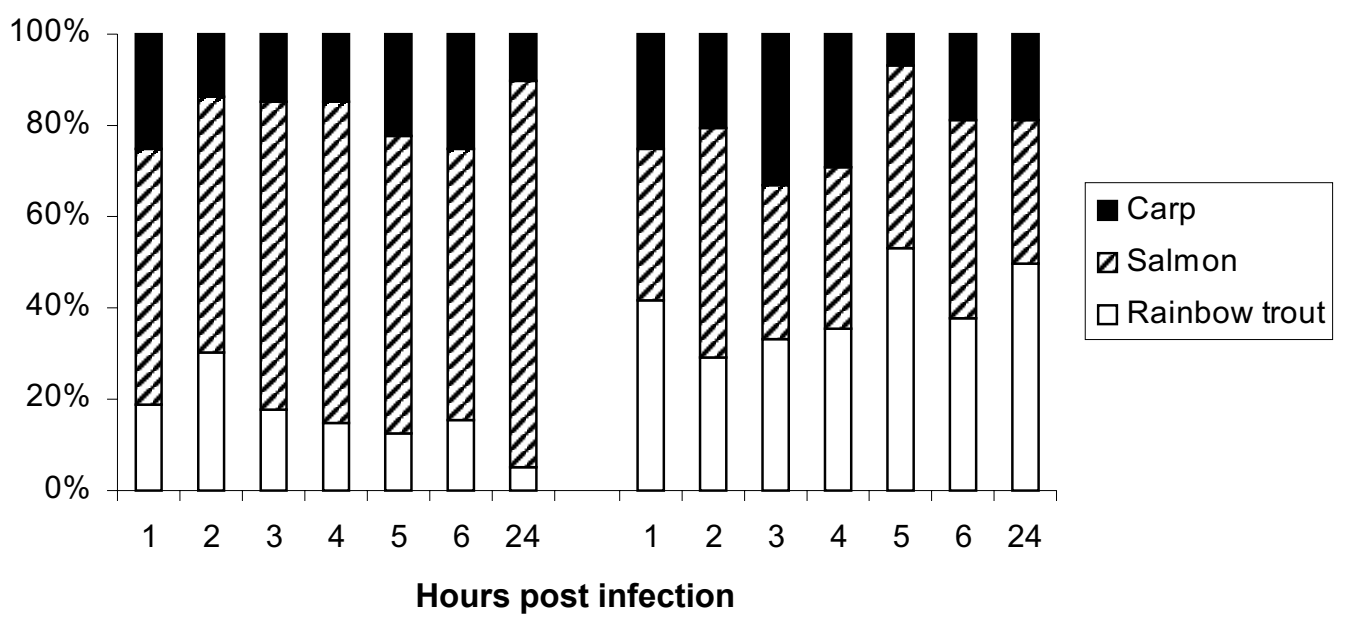

Fig. 1. In vitro colonisation by Gyrodactylus salaris (left) or Gyrodactylus derjavini (right) of fish scales (salmon, rainbow trout, carp); one scale type per exposure; within $24 \mathrm{~h}$; scale-attached parasites only; relative percentage of parasites on a specific scale type in relation to all attached parasites is shown; each column represents a summary of 9 Petri dishes

\section{DISCUSSION}

The results of the present in vitro work, compared to previous in vivo experiments (Buchmann et al. 2004) indicate, that both $G$. derjavini and G. salaris are less efficient in their colonisation of host material (scales) in vitro. A possible explanation could be a decrease of host substances released from the scales due to the preincubation period of $24 \mathrm{~h}$. Further, the artificial environment including tissue culture medium and antibiotics could also be suggested to influence the host findings. Finally, the worms could be partly dependent on host movements to obtain a higher host colonisation rate. However, despite this low colonisation rate, it was in fact noted that the par- asites are able to discern in vitro to some extent between epithelium-covered scales from different fish species. Thus, G. derjavini preferentially colonised epithelia from rainbow trout and G. salaris mainly relocated to scales from salmon. Scales from an atypical host (carp) became colonised at a significantly lower rate. This corresponds to the observations, conducted in vivo by Buchmann et al. (2004), where G. salaris and G. derjavini showed predilection for live representatives of Atlantic salmon and rainbow trout, respectively. Brown trout, $S$. trutta, is the natural host for G. derjavini while rainbow trout, O. mykiss, is a host once introduced to Europe, where G. derjavini is endemic. However, it has been shown that rainbow trout

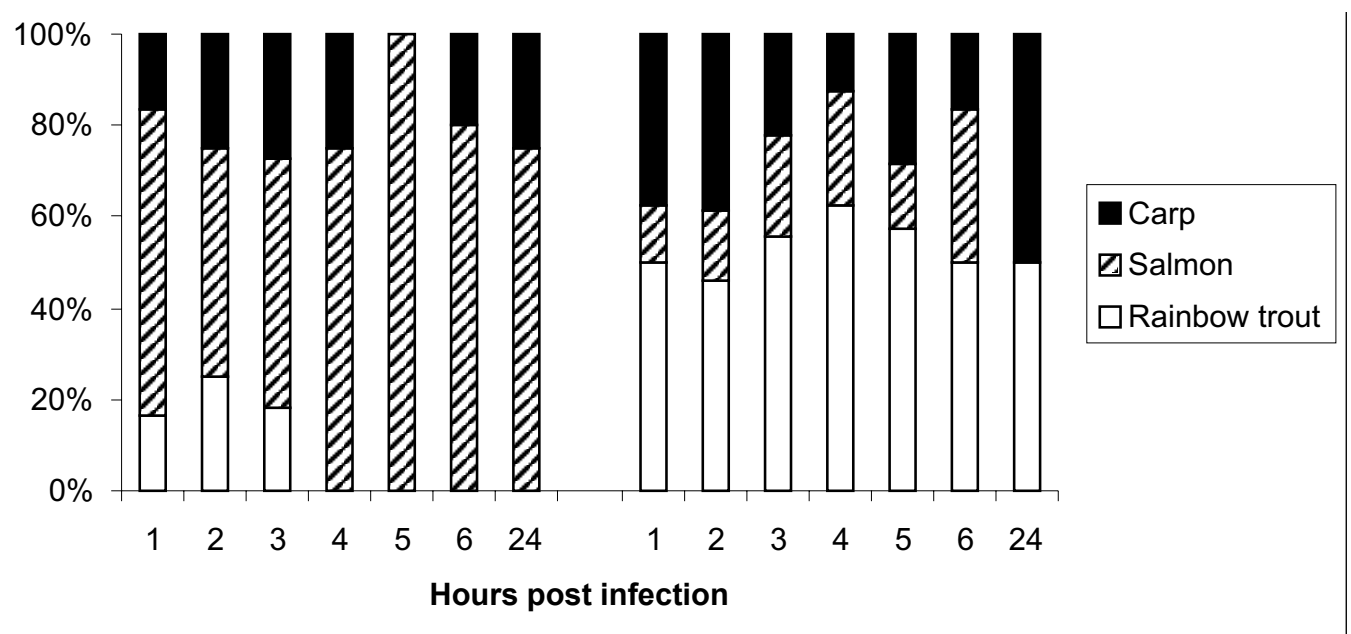

Fig. 2. In vitro preference by Gyrodactylus salaris (left) or Gyrodactylus derjavini (right) of fish scales when simultaneously exposed to three scale types (salmon, rainbow trout, carp), in one Petri dish, within $24 \mathrm{~h}$; scale-attached parasites only; relative percentage of parasites on a specific scale type in relation to all attached parasites is shown; each column represents a summary of 10 Petri dishes 
exhibits comparable interactions with this parasite (Buchmann and Uldal 1997) and rainbow trout/G. derjavini system is a suitable laboratory model for a susceptible but eventually responding host. Due to lack of totally pathogen-free specimens of $S$. trutta we decided to use rainbow trout material in the present investigation. During the colonisation experiments it was noted in several cases that the total number of parasites in the Petri dishes decreased due to some parasite mortality. However, some of the live parasites were bearing developed embryos in their uteri, which subsequently were born in vitro. Therefore, the total number fluctuated, increasing at five occasions.

Studies on monogenean host selection in vitro were previously conducted by Kearn (1967), who showed that oncomiracidia of Entobdella soleae preferred scales of its natural host common sole, Solea solea (L.), over those of many other species. This will add to the notion that the host specificity of monogeneans is, to some part, associated with a recognition system between the host and the parasite.

It is likely that host behaviour (movements) influenced the attachment of parasites in the in vivo study described by Buchmann et al. (2004), by increasing the chances of direct contact between host and parasite. In the present in vitro work, however, only isolated cell-covered scales were used. This indicates that the stimuli, initiating the observed host-specific colonisation, are connected with factors present in the scales. Although, it cannot be excluded that gyrodactylids recognise the specific host cell micro-architecture (Buchmann and Bresciani 1998), a likely explanation is that the scales covered with epidermal tissue are producing substances, which can be detected by the host-specific parasite. Monogeneans, in general, are known to possess sensory organs (Lyons 1969 , Watson and Rohde 1994) and have been suggested to involve lectin-carbohydrate recognition (Buchmann 2001, Yoshinaga et al. 2000, 2002, Jørndrup and Buchmann 2005), pH-related interactions (Hirazawa et al. 2003) and other compounds (Whittington et al. 2000) in their communication with their microhabitat in the fish. Host movements are probably necessary to secure parasite-host contacts whereby these short-range chemo-attractants can play a role. In fact, the total colonisation rate experienced in this in vitro system $(<34 \%)$ was much lower compared to in vivo colonisation rates $(>80 \%)$ experienced by Buchmann et al. (2004). This will stress that chemoattraction is not the only important factor when gyrodactylids are colonising their hosts. In order to elucidate to what extent host movements are responsible for the additional host colonisation a series of studies should be conducted with live but immobilised host.

\section{ACKNOWLEDGEMENTS}

This work was part of the EU-project QLRT-200001631 "The Genetic Basis for Gyrodactylus salaris resistance in Atlantic salmon (Salmo salar)" and is associated with the Research School and Network SCOFDA supported by the Danish Agricultural and Veterinary Research
Council. The authors are indebted to Dr. Tor Atle Mo, National Veterinary Institute, Oslo, Norway, for providing the Gyrodactylus salaris strain from the river Laerdalselva in Norway. Dr. Geert Wiegertjes, Wageningen University, The Netherlands, kindly delivered carp fry for the experiments.

\section{REFERENCES}

Bakke T.A., Harris P.D., Cable J. 2002. Host specificity dynamics: observations on gyrodactylid monogeneans. International Journal for Parasitology 32: 281-308.

Buchmann K. 2001. Lectins in fish skin: do they play a role in monogenean-fish host interactions? Journal of Helminthology 75: 227-232.

Buchmann K., Bresciani J. 1998. Microenvironment of Gyrodactylus derjavini on rainbow trout Oncorhynchus mykiss: Association between mucous cell density in skin and site selection. Parasitology Research 84: 17-24.

Buchmann K., Lindenstrøm T. 2002. Interactions between monogenean parasites and their fish hosts. International Journal for Parasitology 32: 309-319.

Buchmann K., Uldal A. 1997. Gyrodactylus derjavini infections in four salmonids: Comparative host susceptibility and site selection of parasites. Diseases of Aquatic Organisms 28: 13-22.

Buchmann K., Madsen K.K., Dalgaard M.B. 2004. Homing of Gyrodactylus salaris and G. derjavini (Monogenea) on different hosts and response post-attachment. Folia Parasitologica 51: 263-267.

Hirazawa N., Oshima S., Mitsuboshi T., Yamashita S. 2003. Mucus $\mathrm{pH}$ of the tiger puffer Takifugu rubripes is an important factor for host identification by the monogenean Heterobothrium okamotoi. Parasitology 127: 225-230.

Jørndrup S., Buchmann K. 2005. Carbohydrate localization on Gyrodactylus salaris and G. derjavini and corresponding carbohydrate binding capacity of their hosts Salmo salar and S. trutta. Journal of Helminthology 79: 1-6.

Kearn G. 1967. Experiments on host-finding and hostspecificity in the monogenean skin parasite Entobdella soleae. Parasitology 57: 585-605.

Lyons K.M. 1969. Sense organs of monogenean skin parasites ending in a typical cilium. Parasitology 59: 611-623.

Malmberg G. 1993. Gyrodactylidae et gyrodactylose des salmonidae. Bulletin Français de la Pêche et de la Pisciculture 328: 5-46.

Mo T.A. 1991. Seasonal variations of opisthaptoral hard parts of Gyrodactylus salaris Malmberg, 1957 (Monogenea: Gyrodactylidae) on parr of Atlantic salmon Salmo salar L. in laboratory experiments. Systematic Parasitology 20: 11-20.

Mo T.A. 1993. Seasonal variations of opisthaptoral hard parts of Gyrodactylus derjavini Mikailov, 1975 (Monogenea: Gyrodactylidae) on brown trout Salmo trut- 
ta L. parr and of Atlantic salmon Salmo salar L. parr in the river Sandvikselva, Norway. Systematic Parasitology 26: 225-231.

Mo T.A. 1997. Seasonal occurrence of Gyrodactylus derjavini (Monogenea) on brown trout, Salmo trutta, and Atlantic salmon, $S$. salar, in the Sandvikselva river, Norway. Journal of Parasitology 83: 1025-1029.

Watson N.A., Rohde K. 1994. Two new sensory receptors in Gyrodactylus sp. (Platyhelminthes, Monogenea, Monopisthocotylea). Parasitology Research 80: $442-445$.

Whittington I.D., Cribb B.W., Hamwood T.E., Halliday J.A. 2000. Host specificity of monogenean (platyhelminth) parasites: a role for anterior adhesive areas? International Journal for Parasitology 30: 305-320.
Yoshinaga T., Nagakura T., Ogawa K., Fukuda Y., Wakabayashi H. 2002. Attachment-inducing capacities of fish skin epithelial extracts on oncomiracidia of Benedenia seriolae (Monogenea: Capsalidae). International Journal for Parasitology 32: 381-384.

Yoshinaga T., Nagakura T., Ogawa K., Wakabayashi H. 2000. Attachment-inducing capacities of fish tissue extract on oncomiracidia of Neobenedenia girellae (Monogenea, Capsalidae). Journal of Parasitology 86: 214-219.

Received: 29 November 2006

Accepted: 11 December 2007 Published electronically: 6 February 2007 